\title{
MAXIMIZING SPECTRAL RADII OF UNIFORM HYPERGRAPHS WITH FEW EDGES
}

\author{
Yi-Zheng Fan ${ }^{1 *}$, Ying-Ying Tan ${ }^{1,2}$, XI-XI Peng $^{1}$ \\ AND \\ AN-Hong LiU ${ }^{1}$ \\ ${ }^{1}$ School of Mathematical Sciences, Anhui University \\ Hefei 230601, P.R. China \\ ${ }^{2}$ School of Mathematics \& Physics, Anhui Jianzhu University \\ Hefei 230601, P.R. China \\ e-mail: fanyz@ahu.edu.cn
}

\begin{abstract}
In this paper we investigate the hypergraphs whose spectral radii attain the maximum among all uniform hypergraphs with given number of edges. In particular we characterize the hypergraph(s) with maximum spectral radius over all unicyclic hypergraphs, linear or power unicyclic hypergraphs with given girth, linear or power bicyclic hypergraphs, respectively.
\end{abstract}

Keywords: tensor, spectral radius, unicyclic hypergraph, bicyclic hypergraph, girth.

2010 Mathematics Subject Classification: 05C65, 15A18, 15A69.

\section{REFERENCES}

[1] C. Berge, Graphs and Hypergraphs (North-Holland, New York-Amsterdam-Oxford, 1976).

[2] A. Bretto, Hypergraph Theory: An Introduction (Springer, Chambridge-HeidelbergNew York-Dordrecht-London, 2013).

[3] R.A. Brualdi and A.J. Hoffman, On the spectral radius of (0,1)-matrices, Linear Algebra Appl. 65 (1985) 133-146. doi:10.1016/0024-3795(85)90092-8

\footnotetext{
${ }^{*}$ Corresponding author.
} 
[4] R.A. Brualdi and E.S. Solheid, On the spectral radius of connected graphs, Publ. Inst. Math. (Beogard) (N.S.) 39 (1986) 45-54.

[5] K.C. Chang, K. Pearson and T. Zhang, Perron-Frobenius theorem for nonnegative tensors, Commun. Math. Sci. 6 (2008) 507-520.

doi:10.4310/CMS.2008.v6.n2.a12

[6] K.C. Chang, K. Pearson and T. Zhang, On eigenvalue problems of real symmetric tensors, J. Math. Anal. Appl. 350 (2009) 416-422. doi:10.1016/j.jmaa.2008.09.067

[7] L. Collatz and U. Sinogowitz, Spektren endlicher Grafen, Abh. Math. Sem. Univ. Hamburg 21 (1957) 63-77. doi:10.1007/BF02941924

[8] J. Cooper and A. Dutle, Spectra of uniform hypergraphs, Linear Algebra Appl. 436 (2012) 3268-3292. doi:10.1016/j.laa.2011.11.018

[9] S. Friedland, S. Gaubert and L. Han, Perron-Frobenius theorem for nonnegative multilinear forms and extensions, Linear Algebra Appl. 438 (2013) 738-749. doi:10.1016/j.laa.2011.02.042

[10] Y. Hong, On the spectra of unicyclic graph, J. East China Norm. Univ. Natur. Sci. Ed. 1 (1986) 31-34.

[11] S. Hu, L. Qi and J.-Y. Shao, Cored hypergraphs, power hypergraphs and their Laplacian H-eigenvalues, Linear Algebra Appl. 439 (2013) 2980-2998. doi:10.1016/j.laa.2013.08.028

[12] H. Li, J.-Y. Shao and L. Qi, The extremal spectral radii of k-uniform supertrees, J. Comb. Optim. (2015), in press. doi:10.1007/s10878-015-9896-4

[13] R. Merris, Degree maximal graphs are Laplacian integral, Linear Algebra Appl. 199 (1994) 381-389. doi:10.1016/0024-3795(94)90361-1

[14] D.D. Olesky, A. Roy and P. van den Driessche, Maximal graphs and graphs with maximal spectral radius, Linear Algebra Appl. 346 (2002) 109-130. doi:10.1016/S0024-3795(01)00504-3

[15] K. Pearson and T. Zhang, On spectral hypergraph theory of the adjacency tensor, Graphs Combin. 30 (2014) 1233-1248. doi:10.1007/s00373-013-1340-x

[16] L. Qi, Eigenvalues of a real supersymmetric tensor, J. Symbolic Comput. 40 (2005) 1302-1324. doi:10.1016/j.jsc.2005.05.007

[17] P. Rowlinson, On the maximal index of graphs with a prescribed number of edges, Linear Algebra Appl. 110 (1988) 43-53. doi:10.1016/0024-3795(83)90131-3 
[18] Y. Yang and Q. Yang, Further results for Perron-Frobenius theorem for nonnegative tensors, SIAM J. Matrix Anal. Appl. 31 (2010) 2517-2530. doi:10.1137/090778766

[19] J. Zhou, L. Sun, W. Wang and C. Bu, Some spectral properties of uniform hypergraphs, Electron. J. Combin. 21 (2014) \#P4.24.

Received 3 August 2015

Revised 23 December 2015

Accepted 23 December 2015 